\title{
Meary Angle for the prediction of mitral valve prolapse risk in non-syndromic patients with pes planus, a cross-sectional study
}

Antoine Fakhry Abdelmassih ( $\square$ antoine.abdelmassih@kasralainy.edu.eg )

Cairo University Kasr Alainy Faculty of Medicine https://orcid.org/0000-0001-8876-3229

\section{Rafeef Hozaien}

Cairo University Kasr Alainy Faculty of Medicine

Fady Mishriky

Cairo University Kasr Alainy Faculty of Medicine

Mark Michael

Cairo University Kasr Alainy Faculty of Medicine

Mostafa AmanAllah

Cairo University Kasr Alainy Faculty of Medicine

Nada Ali

Cairo University Kasr Alainy Faculty of Medicine

Nadine ElGamal

Cairo University Kasr Alainy Faculty of Medicine

Omar Medhat

Cairo University Kasr Alainy Faculty of Medicine

Mona Kamel

Cairo University Kasr Alainy Faculty of Medicine

Rasha Helmy

Cairo University Kasr Alainy Faculty of Medicine

Mai Sarhan

Cairo University Kasr Alainy Faculty of Medicine

Hams Attalla

Cairo University Kasr Alainy Faculty of Medicine

Omneya Dawoud

Cairo University Kasr Alainy Faculty of Medicine

Athar Marwan

Cairo University Kasr Alainy Faculty of Medicine

Mohamed Ghobashy

Cairo University Kasr Alainy Faculty of Medicine 


\section{Research Article}

Keywords: MVP, Pes Planus, Meary Angle

Posted Date: February 21st, 2022

DOI: https://doi.org/10.21203/rs.3.rs-1352778/v1

License: (c) (i) This work is licensed under a Creative Commons Attribution 4.0 International License. Read Full License 


\section{Abstract}

Objectives: Mitral Valve prolapse (MVP) is the commonest valvular abnormality. There is a reported association between pes planus (PP) and MVP in some syndromes such as Marfan, however, this association has not been tested in non-syndromic cases. The primary outcome of this study is to measure the prevalence of MVP in a population of patients with PP. The secondary outcome parameter is to determine if the Meary angle (MA), a measure of severity of flat foot, can be effectively used in the prediction of the presence of MVP. Forty-One patients with PP have been screened with lateral x-ray foot to determine MA and echocardiography to determine the presence and grade of MVP.

Results: $88 \%$ of screened patients were diagnosed with MVP. MA was correlated with the grade of MVP and showed high diagnostic accuracy (Sensitivity $100 \%$ and Specificity $90 \%$ ) in predicting MVP risk when higher than 5. Children with PP are at a higher risk of MVP than the general population. Utilizing MA in such a specific population for the determination of patients at the higher need for echocardiography seems to be a useful strategy to diagnose MVP in those patients.

\section{Introduction}

Mitral valve prolapse (MVP) is a myxomatous degeneration of the mitral valve, characterized by the displacement of the abnormally thickened mitral valve leaflet into the left atrium during systole. Though generally sporadic, MVP is also associated with a variety of congenital disorders of connective tissue including Marfan syndrome (MS), Ehler-Danlos, syndrome, osteogenesis imperfecta, dominant cutis laxa pseudoxanthoma elasticum, and MASS syndrome (mitral valve prolapse, aortic root dilatation, skeletal changes, and skin changes)[1]

Pes planus or flat foot is a hallmark of connective tissue disorders. When screened by De Maio and colleagues, the skeletal abnormalities associated with Marfan syndrome was found to be $21 \%$ of the involved study subjects. Moreover, defects in fibrillin or collagen genes have been implicated in the development of flat foot as well as mitral valve prolapse. [2]

Normally, the angle between the midline axis of the talus with the midline axis of the first metatarsal is in line at $0^{\circ}$. This angle is termed Meary's angle (MA). MA is used for the grading of flat foot, where an angle greater than 0 but less than 15 degrees signifies a mild deformity, an angle ranging from 16-30 is considered moderate, while an angle exceeding 30 denotes severe PP. The measurement of MA requires a simple and affordable lateral $x$-ray foot. Despite the established etiology shared between PP and MVP, to our knowledge, no study to date has explored the prevalence of MVP in a population of patients with nonsyndromic PP. Moreover, no study has explored the potential use of MA in the prediction of MVP in patients with flat foot. [3]

The primary outcome parameter of this study is to determine the prevalence of MVP in patients with PP. The secondary outcome parameters include the determination of the cutoff level of MA in the context of MVP prediction, and the relationship between MA and the severity of mitral regurge associated with MVP. 


\section{Methods}

\section{Study Subjects}

This study was designed as a cross-sectional study. All patients coming to the outpatient clinic of Cairo University Children Hospital fulfilling the definition of $\mathrm{PP}^{4}$ (the absence of the medial longitudinal arch during weight-bearing) were included in the study. The exclusion criteria comprised of arrhythmia, congenital or acquired heart disease, or any concurrent systemic or connective tissue disease (obesity, diabetes, and systemic lupus erythematosus, MS)

Study Methods

-History taking including age, sex and anthropometric measurements such as weight and height.

-Radiographic assessement of MA

Meary's angle (MA) or talus-first metatarsal angle has been used to identify the severity of PP on lateral weight-bearing foot radiographs.

In the normal weight-bearing foot, the midline axis of the talus is in line with the midline axis of the first metatarsal. That is to say, normally Meary's angle is 0 .

\section{The severity of PP was classified as follows:[3]}

-mild $<15^{\circ}$

-moderate: $16-30^{\circ}$

-severe: $>30^{\circ}$

-Echocardiographic grading of Mitral valve prolapse

Cohen et al grading of mitral valve prolapse has been performed using Cohen et al method as follows: [5]

-Grade I or "mild" prolapse = the maximally prolapsed segment of one or both mitral leaflets or their point of coaptation extended is $5 \mathrm{~mm}$ or less above the plane of the mitral ring in either the parasternal long axis (PSLA) and/or apical four-chamber view. One or both leaflets moved sufficiently above the ring plane to create an echo-free space between the ring plane and the leading edge of the leaflet echoes.

-Grade II or "moderate" prolapse = the maximally prolapsed segment of one or both mitral leaflets or their point of coaptation extended is $>5 \mathrm{~mm}$ above the plane of the mitral ring in either the PSLA and/or apical four-chamber view.

-Grade III or "severe" prolapse = prolapse meeting grade II criteria; in addition, one or both leaflets showed evidence of redundancy defined as the infolding of tissue on itself in any of PSLA or apical view.

\section{Statistical analysis:}


Data was analyzed using IBM\# SPSS\# Statistics version 23 (IBM\# Corp, Armonk, NY, USA). Numerical variables were presented as mean and SD, while categorical variables were expressed as numbers and percentages. (Table 1)

Table 1

Characteristics of the study subjects:

\begin{tabular}{|ll|}
\hline Sex $(n / \%)$ & $\begin{array}{l}\text { Male }(n=22,53.7 \%) \\
\text { Female }(n=19,46.3 \%)\end{array}$ \\
\hline Age $($ Mean \pm SD $)$ & $8.2 \pm 0.9$ \\
\hline Weight (Mean \pm SD) & $24 \pm 5$ \\
\hline MVP Prevalence $(n / \%)$ & $88 \%(n=32)$ \\
\hline MVP grades $(n / \%)$ & No MVP $(n=9,22 \%)$ \\
& MVP grade I $(n=11,27 \%)$ \\
& MVP grade II $(n=16,39 \%)$ \\
& MVP grade III $(n=5,12 \%)$ \\
\hline MA (Mean \pm SD) & $9 \pm 4$ \\
\hline Abbreviations: MVP: Mitral Valve Prolapse, MA: Meary Angle \\
\hline
\end{tabular}

Univariate regression analysis was performed and illustrated as a scatter plot to express the relationship between MA and MVP grade (Fig. 1)

Receiver-operating characteristic (ROC) curve analysis was performed and illustrated as an interactive dot diagram to show the diagnostic accuracy of MA in predicting the risk of MVP in children with flat foot.

(Fig. 2)

P-Value $<0.05$ was considered statistically significant.

\section{Results}

Table 1 shows the characteristics of the study subjects. A high prevalence of MVP was found in patients with PP (88\%). The mean age of our patients was $8.2 \pm 0.9$, which excludes the entity of transient pes planus that usually disappears before the age of six years.

Figure 1 is a scatter plot representing the tight relationship between severity measure of PP, namely MA and MVP grade, with a $P$ value of $<0.001$ and $R$ of 0.91 .

Given this tight relationship, we performed a ROC analysis to determine the sensitivity and specificity of MA in determining the risk of MVP. MA achieved a high sensitivity of $100 \%$, and specificity in predicting the risk of MVP as shown in the interactive dot diagram of Fig. 2. 


\section{Discussion}

Despite its low abundance in the bone matrix, it is increasingly recognized that FBN-1 or Fibrillin is a core player in the pathway of bone formation. [6] Fibrillin plays multiple roles in providing the architectural support necessary for bone formation, which is consistent with the array of skeletal manifestations associated with Marfan syndrome and its variants such as MAAS (Mitral valve prolapse, Aortic root diameter at upper limits of normal for body size, stretch marks of the skin, and Skeletal conditions similar to Marfan syndrome).

Fibrillin also plays an important role in the pathogenesis of cardiovascular disorders as it is an important orchestrator of the meshwork of heart valves notably mitral and aortic valves. Salient evidence for this role is the mitral and aortic anomalies associated with Marfan syndrome. Nevertheless, Fibrillin has been recently linked to the pathogenesis of early aortic stiffness associated with metabolic syndrome. Despite the obvious fibrillin linkage between the cardiovascular and skeletal systems, no studies to date have explored the prevalence of mitral valve prolapse among non-syndromic patients with pes planus; to our knowledge. Instead, all the studies have focused on their association in the context of MS and its variants. $[7,8]$

Our study notes an unexpectedly high prevalence of MVP in patients with flat foot (88\%). This association suggests a common genetic background between both anomalies. This goes in agreement with Raj and Kiel's report about the prevalence of PP and its genetic background where MVP prevalence in the general population ranging from $1-37 \%$. [4]

There is a reported $2.5 \%$ risk of sudden death with mitral valve prolapse, mostly due to associated Long QT syndrome. There are also speculations that tension on the chordae and subvalvular apparatus caused by the prolapsing valve leads to myocardial degeneration and fibrosis, with the subsequent creation of a substrate for arrhythmias. The mentioned risk has urged a notable need for the development of a screening policy of MVP. Three generational screening of families in case of a diagnosed family member with MVP has been recently proposed but the cost-effectiveness of this strategy remains elusive. $[9,10]$

Alternatively, our study offers a new screening policy for MVP, where we suggest the determination of MA in patients with PP. MA angle in lateral foot xray is a routine procedure in such type of patients and will not add a non-required investigation among them. An angle greater than 5 degrees, according to our findings, has a $100 \%$ sensitivity in pointing to the concurrent presence of MVP; which is a justified diagnostic accuracy for performing echocardiography in this high-risk group.

To conclude, MVP is the commonest valvular abnormality. Though benign, a non-negligible risk of arrhythmias and sudden death ensues. This risk warrants the importance of screening patients for this cardiac anomaly. Family history of MVP did not reach a consensus as an enough reason to screen members of the same family. In our study a higher prevalence of MVP has been encountered in patients with flat foot, suggesting a common body of etiology between them. Moreover, the measure of severity of 
PP, namely MA, can be used as a screening tool to determine which patients necessitate echocardiography to diagnose MVP.

\section{Limitations}

-The main limitation to generalize the results of this study is the relatively small sample size. Larger sample sizes are needed to consolidate the results.

-Another limitation was the absence of genetic screening for fibrillin mutations in the study subjects. We are planning to study the genetic background of the association between pes-planus and mitral valve proplapse, away from the classic Marfan-causing mutations.

\section{Declarations}

Ethics approval and consent to participate

The authors assert that all procedures contributing to this work comply with the ethical standards of the Egyptian national guidelines on human experimentation with the Helsinki Declaration of 1975, as revised in 2008 , and has been approved by the institutional committee of Cairo University under the approval number I-2019-322

Accordingly, a written informed consent was taken from all study participants.

Consent for publication

Not Applicable

Availability of data and material

Available upon request

Competing interests

The authors declare that they have no competing interests

Funding

This research received no specific grant from any funding agency, commercial or notfor-profit sectors

Authors' contributions

Antoine AbdelMassih contributed in the conception of the idea, all authors contributed equally to drafting and revision of the manuscript. All authors read and approved the final manuscript 


\section{Acknowledgements}

To our families who are bearing the weight of our sacrifice of time to our patients. If our families were not understanding to the depth of our struggle, we would have never been able to keep the same level of dedication to our patients. To our students that we involve in each step of our research to make them flourish in this field and take the lead the soonest the possible.

\section{References}

1. Weyman AE, Scherrer-Crosbie M. Marfan syndrome and mitral valve prolapse. J Clin Invest. 2004;114:1543-6.

2. De Maio F, Fichera A, De Luna V, Mancini F, Caterini R Orthopaedic Aspects of Marfan Syndrome: The Experience of a Referral Center for Diagnosis of Rare Diseases. Adv. Orthop. 2016, (2016).

3. Flores DV, Gómez CM, Hernando MF, Davis MA, Pathria MN. Adult acquired flatfoot deformity: Anatomy, biomechanics, staging, and imaging findings. Radiographics. 2019. doi:10.1148/rg.2019190046.

4. Raj MA, Tafti D, Kiel J Pes Planus (Flat Feet). StatPearls (2020).

5. Cohen IS. Two-dimensional echocardiographic mitral valve prolapse: Evidence for a relationship of echocardiographic morphology to clinical findings and to mitral annular size. Am Heart $\mathrm{J}$. 1987;113:859-68.

6. Smaldone S, Ramirez F. Fibrillin microfibrils in bone physiology. Matrix Biol. 2016;52-54:191-7.

7. Medley TL, et al. Fibrillin-1 genotype is associated with aortic stiffness and disease severity in patients with coronary artery disease. Circulation. 2002;105:810-5.

8. Rippe M, et al. Mitral valve prolapse syndrome and MASS phenotype: Stability of aortic dilatation but progression of mitral valve prolapse. IJC Hear Vasc. 2016;10:39-46.

9. Cowan MD, Fye WB. Prevalence of QTc prolongation in women with mitral valve prolapse. Am J Cardiol. 1989;63:133-4.

10. Zouridakis EG, Parthenakis FI, Kochiadakis GE, Kanoupakis EM, Vardas PE. QT dispersion in patients with mitral valve prolapse is related to the echocardiographic degree of the prolapse and mitral leaflet thickness. Europace. 2001;3:292-8.

\section{Figures}




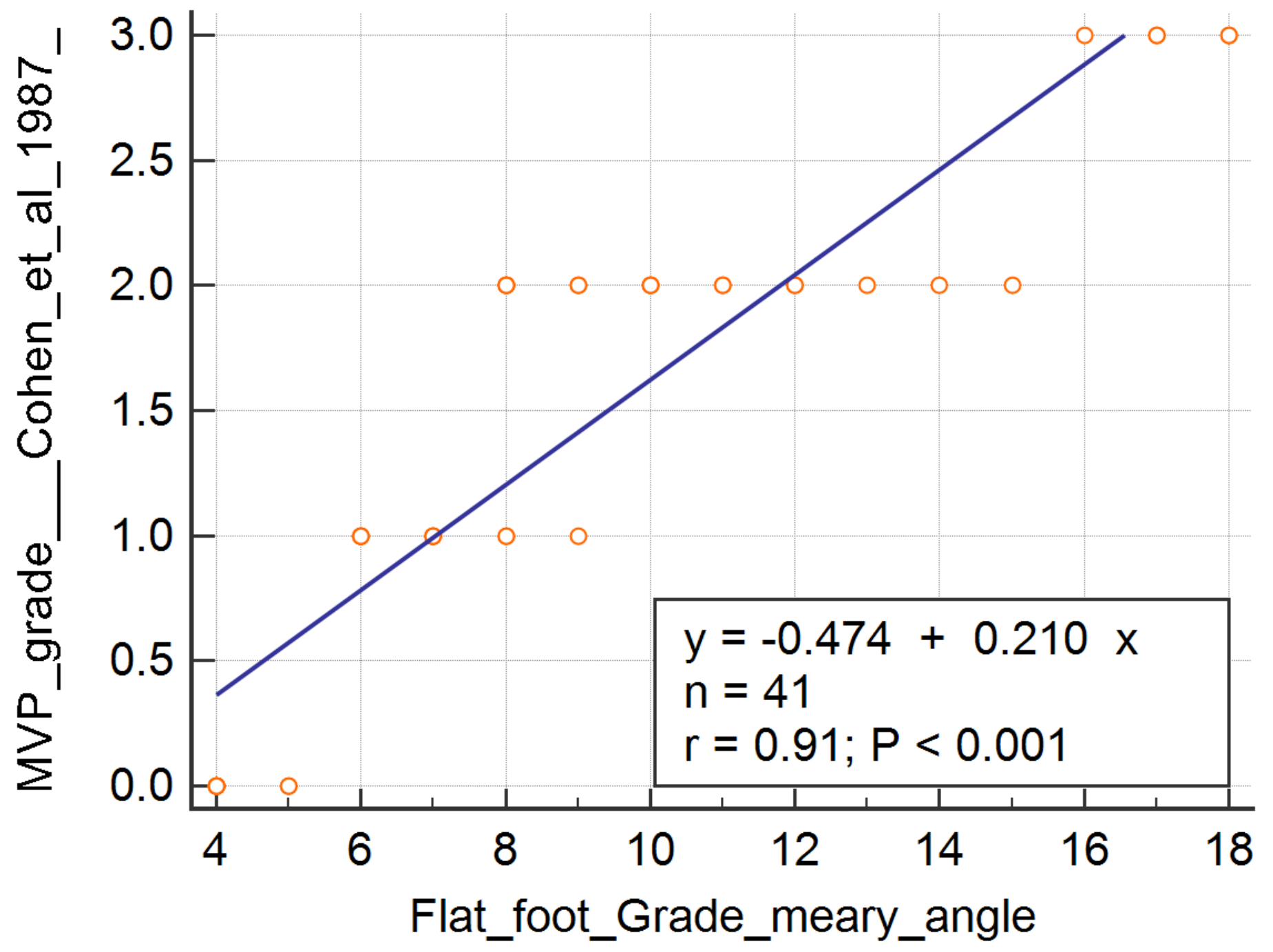

Figure 1

Scatter plot for the correlation between MVP grade and Meary Angle Abbreviations: MVP: Mitral Valve prolapse 


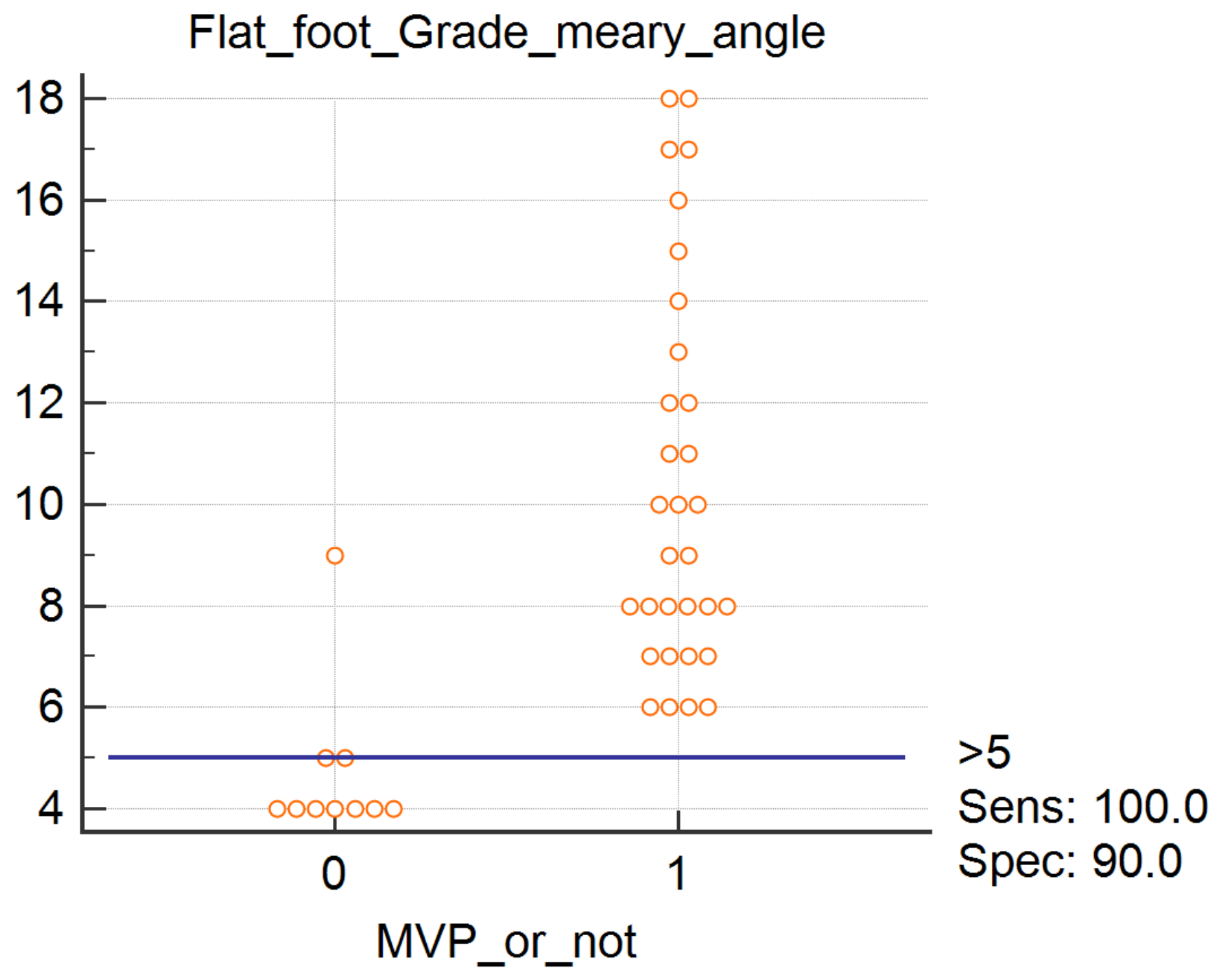

Figure 2

Interactive dot diagram to show the diagnostic accuracy of Meary angle in predicting MVP Abbreviations: $Y$ axis: Meary Angle, $X$ axis: MVP: Mitral valve prolapse (0: Absent/1: Present) Sens: Sensitivity, Spec: Specificity

\section{Supplementary Files}

This is a list of supplementary files associated with this preprint. Click to download.

- Figure3.tif 